\title{
Procesos de Comprensión del Discurso y Adquisición de Segundas Lenguas: ¿Hasta qué Punto Estamos ante una Nueva Variedad de Relativismo Linguiístico?
}

\section{The Effects of Type of Discourse and of a Second Language on Comprehension Processes: A New Linguistic Relativism?}

\author{
José A. León \\ Universidad Autónoma de Madrid \\ Carnegie Mellon University
}

\author{
Inmaculada Escudero \\ UNED \\ Carnegie Mellon University
}

\author{
Augusto Buchweitz \\ Carnegie Mellon University
}

Resumen. A través de este trabajo se examinan diversas cuestiones relacionadas con los procesos de comprensión del discurso escrito y de la adquisición de segundas lenguas como el español, inglés o portugués. El objetivo fundamental de este artículo es tratar de desvelar si el procesamiento que realiza el lector durante la comprensión de un discurso determinado o ante la adquisición de una segunda lengua requiere de una actividad cognitiva diferente a cuando se trata de comprender otro de naturaleza diferente o bajo una lengua diferente. Para ello se analiza por un lado, las diferencias existentes entre diferentes tipos de texto, centrándonos fundamentalmente en el narrativo y el expositivo, analizando sus repercusiones sobre varios niveles de la cognición humana. Por otro, se analizan algunos estudios sobre la adquisición de diferentes segundas lenguas y sus repercusiones sobre su nivel de activación cerebral utilizando la técnica de fMRI. Por último, se plantea hasta qué punto el relativismo lingüístico influye en los procesos de comprensión y de la realización de inferencias y de la adquisición de una segunda lengua.

Palabras claves: relativismo lingüístico, comprensión del discurso, discurso narrativo, discurso expositivo, cognición causal, inferencias, bilingüismo, neuroimagen cerebral, fMRI.

\begin{abstract}
In this paper we address issues related to comprehension processes, and how they can be affected by types of text or written discourse, and by a reader's first or second language. To this end, we discuss the processing of narrative and expository texts, and their effects on different levels of human cognition. Likewise, we discuss the effects of texts written in different codes, that is, in first and second languages. We propose that differences in text comprehension processes occur as a function of their causal nature and of the inferences that are generated during comprehension. In terms of second language comprehension, modern research applying functional magnetic resonance imaging, or fMRI, with bilinguals is discussed in support of the notion that first language comprehension processes are generally replicated in the second language. Therefore, we focus on comprehension processes, generation of interferences, and the processing of a second language as influenced by linguistic relativism.

Key words: linguistic relativism, discourse comprehension, text comprehension, narrative discourse, expository discourse, causal cognition, inferences, bilingualism, L2, causality, functional magnetic resonance imaging, fMRI.
\end{abstract}

La correspondencia sobre este artículo puede dirigirse al primer autor: José Antonio León. Dpto. de Psicología Básica. Facultad de Psicología. Avda Ivan Pavlov, 6. Campus de Cantoblanco. Universidad Autónoma de Madrid. 28049-Madrid (Spain). e-mail: joseantonio. leon@uam.es
Este trabajo ha sido subvencionado por la concesión del Proyecto de Investigación PSI2009-13932 para los años 2010-2013 MCYT. 


\section{Introducción}

Discurso es sinónimo de comunicación. El discurso suele identificarse como un evento comunicativo que se disfraza de múltiples caras según sea el contexto social en el que se desenvuelve (e.g., formal e informal), según su estructura (e.g., narrativo, expositivo), según la intención del comunicador (e.g., persuasivo o pragmático). Todos ellos resultan útiles para comunicarnos. Un aspecto que siempre ha interesado a los lingüistas ha sido el indagar en las características del discurso, sobre sus tipos y formas, sobre sus componentes específicos y distintivos de unos sobre otros. De otro lado, y quizás también debido a la importancia comunicativa del discurso, son muchos los psicólogos cognitivos que se han interesado en su estudio tratando de desvelar qué papel juega el tipo de discurso (especialmente el escrito) en los procesos mentales que están involucrados en la comprensión del mismo o, dicho de otra manera, si los diferentes tipos de discurso requieren también de procesos mentales diferentes. Muchos de estos estudios se han focalizado en el estudio de las inferencias, pues se consideran el núcleo de la comprensión humana y median en toda la actividad mental que tiene que ver con el discurso.

Para los objetivos de este trabajo, la cuestión clave que nos planteamos es analizar precisamente si cada discurso promueve una actividad mental diferente para ser comprendido o, si por el contrario, dicha actividad mental se muestra similar independiente al tipo de discurso presentado. Una respuesta en alguna de estas direcciones nos revelaría importantes aspectos acerca de la naturaleza del discurso, especialmente el escrito, de la justificación "psicológica" de esa variedad de tipos y de importantes aplicaciones educativas que se derivarían de ellas. Por ejemplo, si supusiéramos que los textos narrativos requieren de procesos mentales diferentes a los expositivos, podría deducirse que las inferencias que se generarían de los narrativos serían también distintas, que la estructuración de sus contenidos resultaría también diferente o que la demanda de conocimientos que se requiere podría ser también variada.
Una segunda cuestión que abordaremos también a lo largo de este artículo se sitúa dentro del contexto lingüístico donde se produce el tipo de discurso escrito, lo que nos lleva irremediablemente a reflexionar sobre la influencia del lenguaje en los procesos de comprensión, de la misma manera que ha venido haciendo el relativismo lingüístico sobre el pensamiento. Resulta extraordinariamente importante conocer si estas diferencias, en caso de que se produzcan, puedan deberse a aspectos más relacionados con el funcionamiento de la mente en general, o están de alguna manera tamizados por una influencia lingüística determinada.

Por último, en tercer lugar, analizaremos hasta que punto el procesamiento de una segunda lengua requiere también de procesos mentales diferentes. Ya que este último punto ha sido estudiado muy recientemente desde la activación cerebral, mediante técnicas de neuroimagen cognitiva funcional (functional magnetic resonance imaging - fMRI), revisaremos algunos datos desde esta metodología para argumentar si el bilingüismo requiere de procesos cognitivos similares en la primera y segunda lengua.

\section{Las inferencias como núcleo de la comprensión humana}

Decíamos en la introducción que el discurso es sinónimo de comunicación, y debemos añadir que esta comunicación se realiza cuando el contenido del discurso es comprendido por el receptor. La psicología cognitiva se interesa especialmente por el proceso de comprensión que el lector, oyente, espectador o receptor genera para poder interpretar correctamente un mensaje. Desde un punto de vista psicológico nos resulta importante reconocer que para comprender un discurso el lector construye una representación mental coherente del texto, esto es, que tenga un sentido lógico, un hilo argumental, con el que puede mas tarde interpretar cualquier tipo de discurso. Mientras que esta coherencia no se produzca, la comprensión del discurso no puede com- 
pletarse, como tampoco puede interpretarse correctamente. Ahora bien, no siempre los mensajes o los discursos son completos, más bien al contrario, suelen ser ambiguos, imprecisos, dando por sabido mucha información que se acaba omitiendo. La actividad mental que se produce entonces en el lector es enorme, pues debe procesar no sólo la información que le llega de la fuente del discurso, sino que también la información que el discurso omite y que debe buscarla en el fondo de sus conocimientos. Toda esta actividad mental capaz de conectar la información explicitada en el discurso con la implícita se produce gracias a lo que denominamos inferencias. Ellas son las responsables de establecer la coherencia necesaria de esta representación mental, puesto que rellenan los huecos o lagunas de información que no aparecen de manera explícita en el discurso. El lector construye, al tratar de comprender el mensaje leído, sustituyendo, añadiendo, integrando u omitiendo información del texto. Las inferencias son fundamentales porque tienen un altísimo valor adaptativo para predecir conductas, para entender la realidad, para comprender mensajes abstractos. Gracias a las inferencias podemos desvelar lo "oculto" de un mensaje, leer entre líneas, hacer explícita en nuestra mente la información implícita del pasaje (León, 2003). De una manera general, podríamos afirmar que cualquier información que se extrae del texto y que no está explícitamente expresada en él puede considerarse, de facto, una inferencia. Con ellas generamos expectativas sobre una situación dada y que nos parece cierta, aunque no sea necesariamente así. Las inferencias constituyen el núcleo de la comprensión humana.

La coherencia de la representación mental a la que hemos hecho referencia puede ser tanto local (e.g., cuando conecta la información entre dos frases consecutivas) como global (e.g., conectan segmentos más amplios del discurso). Hace ya algún tiempo que se asume que aquellas inferencias que resultan indispensables para la coherencia local o referencial entre la información más próxima en el texto son generadas durante la comprensión. Ocurre lo mismo para aquellas inferencias que resultan indis- pensables para establecer la coherencia causal local entre información próxima en el discurso. Pero la comprensión de un discurso también necesita de un nivel de coherencia más global. Para construir este nivel más global, el lector reorganiza la información leída dentro de una representación estructurada que, de una manera ideal, consigue integrarla dentro de una estructura global causal. Esto es lo que caracteriza a los modelos que conciben la representación de la comprensión como un modelo mental (JohnsonLaird, 1983), como un modelo de la situación (van Dijk y Kintsch, 1983), como un esquema mental (Schank y Abelson, 1977) o como una red causal (Trabasso, van den Broek y Suh, 1989; van den Broek, 1990). En todos estos casos, las relaciones de coherencia global son principalmente de naturaleza causal, aunque también hay evidencia de relaciones de coherencia espacial dentro de la teoría de los modelos mentales (de Vega, 1995; Glenberg, Meyers y Lindem, 1987; León, 2009).

De manera general asumimos que toda la actividad mental que se genera cuando tratamos de comprender un discurso, sea cual sea su naturaleza, requiere de la activación de inferencias. Ello nos lleva a pensar que las inferencias median en la comprensión del discurso y su estudio resulta idóneo para analizar los procesos mentales que intervienen en la comprensión e interpretación del discurso. De esta manera podemos evaluar la influencia de los tipos de discurso sobre la actividad psicológica que se deriva de ella, de la universalidad o dependencia de la actividad psicológica del contexto cultural o lingüístico donde se produce el discurso, o de su correspondencia neuronal en el cerebro. Estas cuestiones serán planteadas a lo largo de este artículo.

\section{Tipos de discurso}

Tradicionalmente, el discurso escrito ha sido clasificado bajo muchos tipos o categorías, aunque no siempre coincidentes. En general, suele asumirse una clasificación más o menos estándar compuesta por textos descriptivos, expositivos, narrativos, per- 
suasivos y procedimentales (Graesser, 1981) y que se comparte en muchas culturas y lenguas diferentes. Sin embargo, la mayor parte de la investigación suele centrarse fundamentalmente en torno a los textos narrativos y expositivos quizás debido, al menos en parte, a que desde nuestra infancia y durante nuestro proceso educativo nos hemos visto expuestos con mayor frecuencia a este tipo de textos. De ahí que, dada su importancia, les dediquemos una especial atención a estos dos grupos de textos, centrándonos fundamentalmente en el discurso escrito por lo abundante de la investigación realizada hasta este momento.

Texto narrativo. Como es sabido, el discurso escrito narrativo representa contenidos cuya naturaleza suele ser típicamente episódica e incluye un conjunto definido de criterios que comparten semejanzas entre sí. Las narraciones conllevan la caracterización de un personaje y la perspectiva de un protagonista e implican secuencias de hechos o acciones que suelen presentarse en forma de una cadena causal. Esto hace que tenga más sentido hablar de las narraciones en términos de ¿qué ocurrió? en lugar de ¿cuál es la idea principal? A este grupo pertenecen la narración de hechos históricos, autobiografías, historias de ficción, cuentos, fábulas o leyendas. En la medida en que hablamos y analizamos las narraciones, estamos haciendo referencia a una serie de hechos iniciales, episodios, conflictos, estados emocionales, consecuencias, resoluciones y evaluaciones (Labov y Waletzsky, 1967). La mayor parte de las narraciones se orientan por el supuesto de la existencia de una cadena causal que establece la secuencia de eventos, permitiendo al lector (u oyente) alcanzar una interpretación coherente de la historia. Pero, además, la secuencia de eventos posee un orden en el que dichos eventos aparecen en la historia, estableciéndose entre ellas, además de la relación causal, un orden temporal. De esta manera, la narración tampoco podría entenderse sin tener en cuenta la dimensión temporal. De hecho, suele ser el tiempo la principal dimensión de la existencia humana, por lo que todas nuestras acciones están impregnadas de una conciencia temporal. La narra- ción está siempre controlada por el concepto del tiempo y el reconocimiento de que éste es la principal dimensión de nuestra existencia (véase para más detalle Hoerl y McCormack, 2001; Ricoeur, 1998; Rosenbaum y Collyer, 1998).

Según algunos autores, el esquema organizativo de la narración resulta de vital importancia para comprender la actividad humana. Es el esquema que proporciona un propósito y una dirección a nuestros acontecimientos, y hace que nuestras vidas puedan entenderse como un todo. Concebimos nuestra propia conducta y la de los otros dentro de un marco narrativo y, a través de este marco, reconocemos las consecuencias que pueden tener nuestras acciones. Barthes (1966) opina que las narraciones desempeñan funciones sumamente importantes en la vida de las personas. Desde un punto de vista individual, las personas poseen una narración de su propia vida que les permite interpretar o construir lo que son y dónde están situados. Desde un punto de vista social y cultural la narración sirve para dar cohesión a las creencias compartidas y transmitir valores.

Texto expositivo. Por su parte, el discurso escrito expositivo representa aquel conjunto de textos que ofrece conceptualizaciones o formas de construir conocimiento (Kucan y Beck, 1996). Los textos expositivos proporcionan formas de encuadrar nuestro conocimiento del mundo, sintetizar la información procedente de diversas fuentes, clasificar y categorizar este conocimiento en distintas formas jerárquicas (en lugar de estrictamente secuenciales), y representar lo que sabemos en estructuras convencionales de discurso, lo que permite reflejar nuestro conocimiento de manera distinta a como lo hacen las historias o las narraciones. Desde esta perspectiva, resumir las ideas principales de un texto expositivo es una tarea distinta a resumir la secuencia de un argumento, de la misma manera que sintetizar información para construir nuevas relaciones dentro de nuestro conocimiento es una tarea bastante diferente a la de generar inferencias a partir de una historia, o evaluar aspectos de una narración como sus implicaciones morales, el estado emocional que evoca o las acciones del protagonista. 
Los textos expositivos, en oposición a los textos narrativos, se conciben normalmente como fuentes de adquisición de nueva información en diversos dominios o materias tan dispares como las ciencias sociales, las ciencias físicas, las matemáticas o la historia, y en las que incorpora elementos informativos, explicativos o argumentativos, dependiendo del contexto o de la función a la que va dirigido. Estos textos (ya sean libros de texto, páginas web, enciclopedias, etc.) se utilizan habitualmente en aquellas situaciones en las que los estudiantes no poseen un conocimiento previo suficiente, pero donde se espera que este nivel de conocimiento pueda aumentar como resultado de su lectura (véase para mas detalle Goldman y Bisanz, 2002). Quizás uno de los principales inconvenientes que afecta a la discusión que gira en torno a los textos expositivos ha sido reconocer que este tipo de textos no posee marcos organizativos fácilmente perceptibles a través de los cuales presentar la información, bien porque estos marcos organizativos no se han explicitado lo suficiente, o bien el lector no posee estrategias lo suficientemente desarrolladas. Hay, sin embargo, estudios muy precisos que han propuesto esta variable de tipos de discursos en nuevos sistemas de evaluación de la comprensión (e.g. Candel, Fernández y León, 2006; León.2004; León, Escudero, Olmos, Sanz, Dávalos y García, 2009).

\section{Razones para pensar en la existencia de importantes diferencias en el procesamiento cognitivo entre ambos tipos de discurso}

Una cuestión crucial que se viene debatiendo actualmente es conocer hasta qué punto estos dos tipos de discurso se diferencian también en su correlato cognitivo, esto es, si ambos tipos de discurso comparten o no una misma forma de funcionamiento cognitivo, una misma manera de comprender la información contenida en sus páginas, una única forma de generar los mismos tipos de inferencias. Puede pensarse que al tratarse de estructuras y contenidos diferentes, también lo sean todas estas pro- piedades de la cognición. A este respecto, dedicaremos los siguientes apartados.

\section{a) Dos modos de cognición, dos formas de pensar según el tipo de discurso}

Una cuestión que emerge de lo dicho hasta ahora es plantearnos hasta qué punto este conocimiento del discurso (narrativo, expositivo) se corresponde con diferentes tipos de funcionamiento cognitivo y con formas diferentes de establecer relaciones causales. Algunos autores han indagado sobre la existencia de diferentes patrones de funcionamiento cognitivo muy ligados al tipo de relaciones causales que se establecen y también a la forma de organizar la realidad. De entre todos ellos sobresale la aportación de Bruner, quién señaló lo siguiente:

"Existen dos modos de funcionamiento cognitivo, dos maneras de pensar, y cada una proporciona maneras distintas de organizar la experiencia, de construir la realidad. Ambas (a la vez que complementarias) son irreductibles la una a la otra. Los esfuerzos por reducir una a la otra o ignorar una a expensas de la otra conllevan inevitablemente un fracaso en el intento de capturar nuestra rica diversidad de pensamiento... Cada una de estas maneras de conocer tiene sus propios principios operativos y su propio criterio de gramaticalidad. Ambas difieren de forma radical en su manera de proceder." (Bruner, 1986, p. 11).

De acuerdo con Bruner, ambos modos de cognición funcionan de manera distinta, y cada uno utiliza un tipo diferente de causalidad para relacionar los hechos. Para un número importante de autores, estos dos modos de funcionamiento cognitivo correlacionan con dos tipos de textos diferentes: narrativos y expositivos (Brewer, 1980; Einstein, McDaniel, Owen y Coté, 1990; Goldman y Bisanz, 2002; Harris, Rogers y Qualls, 1998; León y Peñalba, 2002; McDaniel, Einstein, Dunay y Cobb, 1986; Polkinghorne, 1988). De hecho, como señala Bruner, estos dos modos de funcionamiento cognitivo se ven reflejados, bien a través de lo que se deno- 
mina una historia estructurada o bien a través de un argumento lógico. Mientras que el modo narrativo busca conexiones particulares entre hechos, el modo lógico-científico, o paradigmático tiende, por el contrario, hacia la búsqueda de condiciones universales y verdaderas. Si el narrativo suele reflejar motivos, acciones y problemas de la vida diaria o de ficción, y está muy influido por la relación temporal que regula la consecución de los distintos hechos o acciones, el modo científico o expositivo, por su parte, trata de cumplir el ideal de "un sistema formal, matemático, de descripción y explicación". Precisamente, los textos científicos se caracterizan por crear conceptualizaciones de ideas, por poseer una organización especificada de manera explícita, por una jerga y terminología específica, y por el uso de términos técnicos (véanse Goldman y Bisanz, 2002; León y Slisko, 2000; Martins, 2002).

$\mathrm{Si}$ tratamos de profundizar en los aspectos que explican las diferencias entre estos dos modos de funcionamiento cognitivo, encontramos que existen varias razones que nos ayudan a entender que los tipos de causalidad implicados en estos dos modos de funcionamiento cognitivo, así como en los dos tipos de texto asociados, son también diferentes (véase Escudero, 2010; Escudero y León, 2007, y León y Peñalba, 2002). El modo lógico o científico tiende hacia la búsqueda de condiciones universalmente verdaderas, predominando una estructura basada en redes causales. Por el contrario, en el modo narrativo, predominan las estructuras basadas en la consecución de objetivos y existen conexiones entre los hechos que no tienen por qué tener estrictamente una base causal. Otra razón posible se basa en el grado de generalización y en el número de observaciones que son necesarias para construir una explicación causal. En la ciencia, el principal objetivo es determinar generalizaciones causales para explicar un conjunto de observaciones (León y Peñalba, 2002). Sin embargo, en la vida diaria, queremos explicar hechos y/o casos aislados o concretos, lo que significa que el método científico, que pone especial énfasis en la generalización, no resulta muy adecuado. Una tercera razón tiene que ver con la estructura de los textos.
Las narraciones orales adquieren la forma de las gramáticas de las historias y su representación mental la del esquema de la historia (Mandler y Johnson, 1977; Stein y Glenn, 1979; Thorndyke, 1977). El conocimiento del esquema de la historia permite al lector desarrollar las siguientes funciones: asociar las ideas que se encuentran dentro de la narración con categorías tales como el escenario, el tema, el argumento y el desenlace (León, 1986; Singer, Harkness y Stewart, 1997); reconocer las partes constituyentes de la historia, como el agente, la intención o el motivo, la situación o el instrumento, que pueden ser considerados como la base de las acciones que se desarrollan (Bruner, 1986; Trabasso, van den Broek y Suh, 1989) e identificar la secuencia temporal de las acciones dentro de un guión (Schank, 1975).

En la mayor parte de las narraciones, la cronología suele ser el principio fundamental de organización causal. Aprendemos que existe la causalidad descubriendo que se da la co-ocurrencia entre las causas y los efectos en el mundo real, así como que las causas preceden a los efectos. Este orden no siempre se sigue en el contexto científico. Los textos expositivos, así como las narraciones complejas, por el contrario, son menos predecibles en su forma que las historias más simples. Se ha sugerido que el mayor nivel de procesamiento de los textos expositivos requiere de categorías abstractas, mecanismos, descripciones y argumentaciones (Otero, León y Graesser, 2002). Estos elementos se organizan en base a estructuras abstractas, tales como cadenas lineales o jerarquías, y en redes retóricas (rhetorical networks), tales como la comparación entre dos o más elementos. Estas dos maneras de entender la causalidad tienen repercusiones importantes en la comprensión de los textos narrativos y expositivos, como veremos en el siguiente apartado.

\section{b) Dos modos diferentes de comprender}

Bajo el supuesto de que existen distintas formas de entender la causalidad, y que éstas quedan reflejadas de algún modo en la formalización de los dis- 
tintos tipos de discurso, autores como Polkinghorne (1988) han llegado a afirmar que diferentes tipos de discurso requieren patrones distintos de comprensión. Por ello, a continuación mostramos algunas de las implicaciones que la causalidad tiene sobre la comprensión de estos dos tipos de texto.

Los lectores comprendemos un hecho cuando somos capaces de relacionarlo con otros hechos dentro del texto. Precisamente, una de las conexiones más importantes, sino la más importante, es la causalidad. No resulta, por tanto, sorprendente que aquellos investigadores que comenzaron a estudiar la comprensión sugirieran que las relaciones causales juegan un papel esencial en la comprensión de las narraciones (Bartlett, 1932; Dewey, 1938; Piaget, 1927 a y b). La investigación sobre la comprensión de la narración durante los años 70 compartía el supuesto de que las representaciones causales eran fundamentales en la comprensión y memoria de los textos narrativos (Mandler y Johnson, 1977; Rumelhart, 1975; Schank y Abelson, 1977; Stein y Glenn, 1979; Thorndyke, 1977). Existían fuertes asociaciones dentro de la memoria entre las acciones de la narración que compartían una relación causal directa (Trabasso y Sperry, 1985). Existe evidencia suficiente que muestra que tanto la fuerza como el número de conexiones causales determinan el nivel de comprensión y el recuerdo de la información leída, así como el grado de importancia que el lector asigna a la información del texto (Trabasso y Sperry, 1985; van den Broek, 1988). Como consecuencia, los modelos causales han prevalecido en el estudio de la comprensión narrativa dentro de la psicología (Graesser, Swamer, Baggett y Sell, 1996; Langston y Trabasso, 1999; Trabasso, Secco y van den Broek, 1984; van den Broek, 1989; van den Broek, Young, Tzeng y Linderholm, 1999).

Con respecto a los textos expositivos, la relación causal, como un principio organizativo básico, es también un principio explicativo que nos habla acerca de 'qué', ‘cómo', 'por qué’ y ‘cuándo’ ocurre la causalidad. Las explicaciones científicas son, por lo general, causales (Salmon, 1998; véase también Ohlsson, 2002) y responden por medio de la interrogación ¿por qué?, esto es, cuando generamos una explicación científica, estamos respondiendo a por qué ocurre un determinado fenómeno. Por ejemplo, es de conocimiento general que la destrucción de la capa de ozono afecta a parte de la fauna y flora y, por tanto, causa serios problemas en la naturaleza. Esta, y otro tipo de creencias, confeccionan la comprensión causal de sentido común del mundo, incluyendo a los seres humanos y sus interacciones con la naturaleza. Las características de este sistema y el modo en que opera es parte del debate científico. Esto es, producimos discurso científico con explicaciones acerca de por qué se produce el deterioro de la capa de ozono, pongamos por caso, sobre cómo, cuándo y donde se produce, así como nos preguntamos sobre cuáles podrían se sus consecuencias.

Varios modelos y teorías dentro de la psicología del discurso se han centrado especialmente en los mecanismos psicológicos que subyacen a la comprensión de las relaciones causales en el contexto científico. Se han llevado a cabo investigaciones sobre las inferencias que explican, elaboran o predicen acciones dentro de la cadena causal en un contexto científico (Britton y Black, 1985; Graesser y Bertus, 1998; Millis y Graesser, 1994; van den Broek, Virtue, Gaddy, Tzeng y Sung, 2002). En ocasiones resulta difícil comprender un texto porque no existe suficiente conocimiento previo sobre el tema, mientras que otras veces se debe a una falta de coherencia. Estas barreras hacen difícil, si no imposible, conectar el texto de manera causal (León, 2004; McKeown, Beck, Sinatra y Loxterman, 1992). Por ello, los textos expositivos requieren de un procesamiento más intenso que los narrativos. La comprensión del discurso científico requiere de tipos diferentes de conocimiento para generar una explicación, tales como un conocimiento abstracto y conceptual, una argumentación lógico-matemática y un conocimiento procedimental o estratégico (Graesser, León y Otero, 2002; León y Peñalba, 2002; León y Slisko, 2000).

Algunos autores han apuntado también diferencias en cuanto al papel que juega la explicación 
según se trate de un tipo de texto u otro (por ejemplo, Polkinghorne, 1988). Las personas normalmente explicamos nuestras propias acciones y las de los demás a través de un argumento. Dentro del esquema narrativo, un hecho puede ser explicado cuando se identifica su papel y su significado dentro de un plan o una meta. Según Polkinghorne, la explicación en la narración es diferente a la del razonamiento lógico-matemático, donde ésta tiene lugar cuando un hecho puede ser identificado como ejemplo de una ley o un patrón de relaciones entre categorías. La explicación a través del uso de leyes proviene de la capacidad para abstraer hechos a partir de contextos particulares y descubrir relaciones que se dan entre un amplio conjunto de ejemplos dentro de una categoría, con independencia del contexto espacial y temporal. Por ello, en principio, uno podría proyectarse hacia atrás o hacia delante en el tiempo, y la relación identificada por el razonamiento formal lógico-matemático podría darse igualmente. Sin embargo, la explicación dentro de la narración depende de un contexto y, por ello, difiere en su forma de la explicación científica. De esta manera, y según Polkinghorne (1988), la simetría que existe entre explicación y predicción en el razonamiento lógico-matemático se rompe en la narración. La explicación narrativa no engloba los sucesos o hechos bajo ninguna ley. La explicación se basa en la aclaración del significado que tienen los hechos ocurridos basándose en la/s consecuencia/s que le siguen. Esto hace que la explicación narrativa, según Polkinghorne, sea retroactiva.

A pesar de estas diferencias, existen algunos estudios que sugieren que la estructura causal en los textos narrativos y expositivos puede no ser tan distinta como parece en un principio (véase León y Peñalba, 2002). Por ejemplo, las estructuras basadas en la consecución de metas, componente importante de los textos narrativos, parece que también se encuentran en algunos textos científicos, tal y como muestran los resultados encontrados por León, Otero, Escudero, Campanario y Pérez (1999). Estos autores encuentran que la identificación de relaciones causales depende, entre otros aspectos, del cono- cimiento previo (por ejemplo, general vs. específico) y del contexto (por ejemplo, físico vs. tecnológico). De esta manera, si el conocimiento es específico, los sujetos se centrarán más en las relaciones causales nucleares, mientras que un conocimiento más general induce con mayor probabilidad una respuesta basada en el sentido común. Por otra parte, mientras que un contexto físico puede facilitar la generación de explicaciones causales basadas en una lógica causal, un contexto tecnológico se centra más en las metas u objetivos.

Conviene, además, hacer una salvedad acerca de las inferencias que son generadas mediante mecanismos que obedecen a principios universales de funcionamiento cognitivo. La realidad nos dice que entre ambos textos predominan tres tipos de inferencias comunes y que corresponden a manifestaciones de la cognición causal y a los procesos comunes y universales de la comprensión. Las primeras se denominan asociaciones y hacen referencia a aquellos conceptos, ideas o ejemplos que los sujetos generan durante la lectura y suelen ser fruto de la activación de su conocimiento previo. Tales inferencias suelen estar basadas en la activación del conocimiento previo del lector y sirven para integrar la información del texto a través de la elaboración de explicaciones o predicciones. Un segundo tipo de inferencia son las inferencias explicativas o antecedentes causales, que aluden a alguna causa, razón o motivo de un determinado acontecimiento y sirven, fundamentalmente, para integrar las distintas oraciones del discurso. Son inferencias hacia atrás, ya que están orientadas hacia la información aparecida previamente en el texto. Por último, las inferencias predictivas se relacionan con la información de las consecuencias causales de un hecho o acción, responden a la pregunta ¿qué pasará después? y pueden implicar expectativas acerca de hechos, objetivos, acciones, resultados o emociones. Este tipo de inferencias se enmarca dentro de las denominadas inferencias hacia adelante. En situaciones "naturales" de comunicación el alcance de estas inferencias puede llegar hasta el $70 \%$ de las que se producen de manera espontánea. 


\section{¿Puede nuestra lengua influir en los procesos de comprensión y a la generación de inferencias de manera distinta a como podría suceder en otra?}

Todo lo señalado hasta ahora apoya la idea de que los tipos de discurso analizados aquí (narrativo y expositivo) y que predominan en las diferentes lenguas y culturas (son, por tanto, transversales), parecen generar diferentes patrones de funcionamiento mental, básicamente centrados en diferentes modos de entender la cognición causal y con ello la forma de pensar. Otra cuestión que se debate al hilo de estas diferencias es la relativa a analizar la universalidad de los procesos cognitivos implicados en la comprensión y en la cognición humana, y si estos son independientes de la lengua que se habla o de si estos procesos mentales son también universales respecto a los dos tipos de discurso aquí analizados. Veremos a continuación el estado de estas cuestiones.

\section{a) La controversia sobre el relativismo lingüistico}

Hoy sabemos que las diferentes lenguas difieren en el modo en que se describe el mundo o la realidad, pero ¿puede el lenguaje que utilizamos y hablamos influir en nuestra manera de pensar? Esta pregunta se ha asociado desde hace muchos años y de manera inevitablemente a Sapir (1921) y Whorf (1956) y al denominado relativismo o determinismo lingüístico. Whorf, por ejemplo, propuso que las categorías y distinciones de cada lenguaje sesga la forma de percibir, analizar y actuar en el mundo. Esta posición teórica de que el lenguaje tiene un impacto en la cognición de sus hablantes es un antiguo y controvertido debate que se ha extendido desde diferentes puntos de vista, especialmente desde la lingüística, psicología y antropología (véase para una revisión Gumperz y Levinson 1996; Lucy 1992). Recientemente, las teorías sobre relativismo lingüístico han resurgido de nuevo (véase Gentner y Goldin-Meadow, 2003, para una revisión).
Las lenguas parecen diferir una de otras, pongamos por caso, en la forma de percibir la distancia, describir emociones o en la manera en que se agrupan los objetos dentro de ciertas categorías gramaticales. Estas diferencias suelen atribuirse a las distintas agrupaciones de las lenguas como, por ejemplo, las germánicas (inglés, alemán, sueco u holandés) de las románicas (español, francés, italiano o portugués) como señalan algunos autores (e.g., Aske, 1989; Slobin, 1996, 1997; Slobin y Hoiting, 1994). Pero de ahí a que una lengua tamice o sesgue de manera general la forma de percibir, analizar y actuar sobre el mundo de manera muy diferente a como se percibe desde otra lengua diferente, se antoja muy lejano. La polémica está servida. Si recientemente desde la perspectiva de los estudios que provienen de la antropología lingüística sugieren importantes diferencias en relación con categorías semánticas en un contexto social como las relaciones de parentesco (e.g., Danziger, 2001; Foley, 1997), desde otra perspectiva diferente, como la que mantienen algunos psicólogos cognitivos y lingüistas, se asume procesos universales y comunes a las diferentes lenguas. De esta manera, lenguas tales como el español, inglés, alemán, japonés o finlandés comparten un mismo substrato mental o neuronal. Como señalan Bates, Devescovi y Wulfeck (2001) "las lenguas no viven en diferentes partes del cerebro". Sobre los estudios realizados hasta ahora sobre este tema desde la psicología, Pinker resume que "no se ha encontrado evidencia científica alguna de que la lengua influya de manera dramática en la manera de pensar de sus hablantes" (1994, p. 57). Bien es verdad que este asunto, aunque ha interesado a algunos psicólogos cognitivos en el pasado, no fue un tema central en el estudio del lenguaje y de la cognición humana.

Una de las razones por la que esta controversia sigue manteniéndose viva hoy día se deba, quizás, a que la investigación sobre el relativismo lingüístico no se ha completado con el estudio de los procesos cognitivos que tienen lugar durante el curso del lenguaje. Slobin (2003) afirma, con mucha razón, que la voluminosa literatura existente sobre 
relativismo lingüístico se ha dirigido fundamentalmente a la búsqueda de esa posible influencia de una lengua particular sobre la cognición en situaciones en las que el lenguaje no está siendo utilizado. Ello representa una larga tradición en la que los antropólogos, psicólogos y lingüistas han buscado relacionar aspectos gramaticales y semánticos de una lengua limitando su esfuerzo, bien al estudio de la palabra, bien a su carácter epistemológico, o bien al contexto cultural a la que pertenece la comunidad de hablantes de esa lengua. A todo esto habría que añadir, además, otro problema relacionado con la metodología utilizada por los investigadores en este campo. Para analizar las diferencias entre lenguas, las diferentes propuestas (dentro de la investigación transcultural) han utilizado diferentes metodologías cuyo uso ha dificultado, en la mayor parte de las veces, una posible comparación entre ellas (véase para una revisión Berry, 2000; Berry, Poortinga, Segall y Dasen, 1992; Escudero, 2004; Kim, Park y Park, 2000). Básicamente, pueden distinguirse dos perspectivas metodológicas que caracterizan los estudios transculturales. Una de ellas considera la lengua como una variable intersubjetiva, de tal manera que cuando se trata de contrastar dos o más lenguas entre sí se aplica el mismo diseño experimental para determinar cómo las diferencias lingüísticas afectan al desarrollo de una determinada tarea. La segunda perspectiva trata la lengua como un experimento en sí mismo, intrasubjetiva, mediante la exploración de propiedades particulares de una determinada lengua con el objeto de formular preguntas que no pueden responderse desde otra lengua. Este hecho hace difícil, cuando no imposible, poder contrastar sus resultados.

\section{b) Algunas propuestas de análisis}

Slobin (1987) sugiere en su hipótesis thinking for speaking (pensar para hablar) que cuando se construyen las declaraciones en el discurso, el hablante fija su pensamiento sobre la forma lingüística dispo- nible. Sin embargo, hay una escasísima investigación previa transcultural que nos ayude a determinar si las diferencias del discurso producen realmente variaciones importantes dentro del mismo contexto. La mayor parte de los estudios comparativos provienen de la lingüística o la psicolingüística, y apenas aportan algunos aspectos relacionados con los procesos de la comprensión de textos. Solo unos pocos estudios han intentado analizar diferencias culturales en el contexto de la tradición oral de cuentos populares (e.g., Chafe, 1980; Tannen, 1980, 1983, 1984, 1988) o en el recuerdo (e.g., Mandler, Scribner, Cole y De Forest, 1980).

Como una propuesta alternativa a la que plantea Slobin hemos sugerido la hipótesis speaking for understanding (hablar para comprender) (Escudero y León, en revisión), que señala que cuando se trata de comprender un discurso, el lector u oyente fija su pensamiento en la forma en que genera inferencias, siendo éstas universales a la cognición humana y muy fuertemente asociadas a la estructura causal del discurso. Este hecho nos permite codificar diversas dimensiones lingüísticas que median en el proceso de comprensión cuando el lector genera inferencias que necesita para comprender un texto, mientras piensa en voz alta. Desde este punto de vista pensamos que es posible comparar efectos anticipatorios y consecuentes relacionados con los procesos cognitivos tales como las inferencias mismas, la comprensión o la interpretación, todos ellos válidos como un interfaz crítico entre el lenguaje y la cognición.

En nuestro trabajo más reciente, hemos tratado de evaluar aquellas declaraciones verbales que realiza el lector cuando trata de describir lo que ha leído de un texto mientras lo comprende, contrastando lenguas diferentes y evaluando su influencia en los procesos de comprensión. Más específicamente, el objetivo del que partíamos en esta línea de investigación era contrastar si en dos lenguas diferentes los tipos de inferencias (asociaciones, explicaciones y predicciones) que se realizan para comprender un determinado tipo de texto se regulan mediante un patrón universal y común o, por el contrario, surgen 
matices suficientes para considerar que la lengua pueda ser influyente en tal proceso. Como forma adicional a este primer objetivo, hemos tratado de explorar estos mismos efectos, pero esta vez centrados en distintos tipos de discurso escrito (fundamentalmente narrativos y expositivos). Hasta el momento, hemos realizado dos estudios, uno en el que se ha contrastado lectores nativos de lengua germánica (inglés, EE.UU.) con lectores provenientes de una lengua románica (español, España) (Escudero y León, en revisión) y un segundo estudio en el que ha comparado la lengua española con la finlandesa (León, Hytönenn y Escudero, en preparación) con resultados muy similares. Finalmente, se esta iniciando un estudio comparando la lengua española con la japonesa.

El patrón de inferencias analizadas en estos dos primeros estudios fue muy similar al comparar los lectores de lengua española e inglesa (Escudero y León, en revisión) como los de lengua española y finlandesa (León et al., en preparación). Esta similitud en los patrones de inferencias relacionados con la comprensión apoya la idea de que la cognición causal se constituye como un principio universal e independiente del relativismo lingüístico, lo que coincide con diversas teorías acerca de la causalidad como han señalado algunos autores (e.g., Morris, Nisbett y Peng, 1995; Sperber, Premack y Premack, 1995). Desde este punto de vista, la cognición causal se asume entonces como una propiedad universal de la mente, capaz de representar regularidades causales o principios generales en la generación de relaciones causales.

Los resultados obtenidos en ambos experimentos revelaron, sin embargo, diferencias significativas en cuanto al tipo de inferencias que se realizaban cuando los sujetos leyeron los distintos tipos de texto. En este sentido, los cuentos y las narraciones generaron mayor número de predicciones respecto a los textos clasificados como expositivos, mientras que éstos evocaron mayor número de explicaciones respecto a los narrativos. Estos datos nos permiten extraer una conclusión importante, y es que cada tipo de texto parece determinar la activación de unas expectativas de lectura distintas y de unos contenidos y procesos específicos. Por otro lado, el análisis de los resultados ofrece un dato importante que tiene que ver básicamente con la forma en la que procesamos los textos narrativos respecto a los expositivos. Según nuestra hipótesis inicial, los textos narrativos se caracterizan por generar un número significativamente mayor de inferencias predictivas en comparación con el resto de los textos, siendo menos frecuentes en los textos expositivos. Sin embargo, las explicaciones fueron significativamente más altas en los textos expositivos.

Estos datos sugieren que la dirección del procesamiento en ambos tipos de textos puede ser distinta. Es decir, los textos expositivos parecen requerir de un mayor esfuerzo de procesamiento, puesto que necesitan generar mayor número de conexiones con la información causal antecedente. Por el contrario, los textos narrativos buscan la información causal consecuente y parecen requerir de mayor esfuerzo a la hora de recuperar los conocimientos almacenados en la memoria a largo plazo. Por otra parte, y de acuerdo con lo esperado, el número de asociaciones fue significativamente mayor en los textos expositivos en comparación con las narraciones. De hecho, podemos decir que, debido al tipo de contenido y estructura que caracteriza a este tipo de textos, las asociaciones están ligadas fuertemente a las explicaciones. Generar una explicación implica, entre otras muchas cosas, activar nuestro conocimiento previo y, por ello, activar conceptos necesarios para producir estas explicaciones.

Nos gustaría, no obstante, matizar este carácter universal de las inferencias. A pesar de la nitidez de los resultados, estos estudios no pueden, por si solos, ser concluyentes. Por ello, este tipo de estudios requiere de un enfoque transcultural más amplio, pudiendo contrastar estos resultados con culturas y/o lenguas más alejadas de la nuestra (por ejemplo, culturas asiáticas, árabes, etc.). Obviamente, esto no está exento de dificultades, pues podría hacer más complejo abordar culturas tan diferentes con los mismos textos. 


\section{¿Puede el hecho de que una determinada lengua afecte a los procesos de comprensión de manera distinta a como podría suceder en otra? El caso del bilingüismo a través de estudio de la resonancia magnética funcional (fMRI)}

Otra propuesta alternativa a la que plantea Slobin y a la planteada por Escudero y León (en revisión), es la derivada de estudios cognitivos que se han centrado en la resonancia magnética funcional (functional Magnetic Resonance Imaging, fMRI) en la que se obtienen imágenes cerebrales. Dedicaremos esta sección a analizar la investigación reciente sobre un ámbito que ha suscitado un enorme interés en los estudios cognitivos en relación con el bilingüismo en diversas lenguas y algunas implicaciones que nos permita comprender mejor como el lenguaje es procesado.

En general, la resonancia magnética funcional, también denominada neuroimagen, imagen cerebral o, simplemente, fMRI, nos proporciona una ventana donde podemos mirar nuestro cerebro por dentro. $\mathrm{La}$ investigación se esfuerza por identificar, describir y analizar cuidadosamente las propiedades funcionales de las diversas regiones cerebrales. Una introducción de las diferentes metodologías, sus ventajas y restricciones queda mas allá de las pretensiones de este articulo, pero puede encontrarse una excelente introducción en Cabeza y Kingstone (2006). La resonancia magnética funcional es, por tanto, una técnica que se utiliza con el fin de desarrollar un mapa sobre la actividad cerebral durante el desarrollo de diferentes tareas, como, por ejemplo, las tareas relacionadas con la comprensión del lenguaje. La investigación de fMRI se basa en las comparaciones de la activación cerebral resultante en diferentes situaciones. Por ejemplo, si a un hablante se le presenta una tarea consistente en leer u oír oraciones en distintas lenguas, podría compararse si la activación cerebral que se deriva de esta tarea es similar o diferente dependiendo de la lengua que este utilizando. De esta manera, podríamos saber más sobre si el relativismo lingüístico repercute en la actividad cerebral, activando unas regiones cerebrales u otras $y$, por ende, podríamos deducir si influye o no en el pensamiento.

Desde un punto de vista cognitivo, cualquiera de los métodos neurocientíficos (entre los que se incluye el fMRI, la tomografía de emisión de positrones, PET, la encefalografía magnética, MEG, y los potenciales cerebrales evocados, ERP), proporcionan una información multidimensional más allá de la que llegan los métodos experimentales u observacionales, accediendo directamente al cerebro. De esta manera y basándose en la misma identificación de las áreas cerebrales, este tipo de estudios también puede ayudar a comprender mejor que procesos mentales están incluidos en diferentes tareas cognitivas. En nuestro caso, nuestro foco de estudio gira en torno de estudios de fMRI en tareas que requieren de leer u oír en distintas lenguas y las posibles implicaciones de sus diferencias (o de la ausencia de ellas) entre el procesamiento de la primera y segunda lengua, como es el caso de hablantes bilingües. Esta es una oportunidad inmejorable para analizar no sólo lo que ocurre en hablantes bilingües cuando leen $\mathrm{u}$ oyen una información determinada en diferentes lenguas, sino también lo que sucede dentro del mismo hablante, dentro de su propia actividad cerebral, cuando lee $\mathrm{u}$ oye la misma información en su lengua materna (en adelante la denominaremos L1) o en su segunda lengua adquirida (en adelante L2).

\section{La imagen cerebral en tareas que demandan el uso de dos lenguas}

La competencia en una segunda lengua y la edad con la que se adquiere son dos factores que nos ayudan a entender algo más sobre la heterogeneidad de la población de bilingües en el mundo. Podríamos decir que existe una mayor o menor competencia bilingüe (o más o menos habilidad respecto al uso que se hace de una segunda lengua), como hay también un bilingüismo temprano o tardío, que frecuentemente se asocia con la edad en la que dicha segunda lengua fue aprendida. De acuerdo con Paradis (2003), aprender una segunda lengua antes de los 
siete años supone un índice claro para poder diferenciar un bilingüismo temprano de otro tardío. Buena parte de la investigación que ha utilizado la imagen cerebral se ha centrado sobre el estudio de estos dos factores (competencia y edad de adquisición) entre la L1 y L2, que pasaremos a describir brevemente.

Cuando se ha investigado grupos de bilingües que difieren en su nivel de competencia respecto a una segunda lengua (competentes vs. menos competentes), así como respecto a la edad en la que esa L2 se ha adquirido (temprana vs. tardía), los resultados muestran claramente que ambos factores influyen en la organización cerebral del procesamiento del lenguaje. Los resultados varían sobre como la competencia y edad de adquisición determina áreas neuronales activadas por la lengua materna (L1) y la segunda lengua (L2), en la medida en que ambas se relacionan con el conocimiento y la habilidad de la L2. Algunos estudios que se han centrado sobre la edad de adquisición (bilingüismo temprano vs. tardío) difiere tanto en la cantidad de activación (Mahendra et al., 2003) como en la localización cerebral donde se produce la activación (Kim et al., 1997; Marian et al., 2007). Tales diferencias en la activación cerebral encontrada bilingües competentes y menos competentes parece depender del conocimiento declarativo que se desarrolla en los estadios más tempranos de la competencia de L2, esto es, en su fase de adquisición.

Hasta cierto punto podemos considerar que estos resultados son congruentes con lo esperado, esto es, que aquellos hablantes bilingües muy competentes en su L2 y que adquirieron dicha lengua muy tempranamente difieran ampliamente de aquellos otros que no son tan competentes, o incluso cuando lo menos competentes en su L2 se comparan con su L1. La cuestión estriba más bien en que es lo que ocurre en el caso de que el hablante sea igualmente competente en L1 y L2, y aunque sea igualmente competente en ambas lenguas difiera en la edad de adquisición, ¿se producirían cambios relevantes en su actividad cerebral? Y que ocurría en el caso en que los bilingües hablasen dos lenguas completamente diferentes (e.g., asiáticas frente a europeas, en los que hay grandes cambios en los sistemas de escritura), ¿se producirían igualmente diferencias en su actividad cerebral? En caso de que las hubiese, se deberían éstas al relativismo lingüístico, al cultural, a los distintos sistemas de escritura, tal y como ocurría en el caso de los tipos de textos narrativos/expositivos, o se debería, quizás, a la demanda de la tarea?

Sobre estos puntos no existe aún suficiente evidencia empírica. Cabe señalar, sin embargo, que los datos de los que se dispone sobre el bilingüismo temprano y tardío en niveles de competencia similares respecto a L1 y a L2, no se ha encontrado diferencias en que la edad de adquisición influyese significativamente en la representación cerebral de L2 (Perani et al., 1998). De hecho, en un estudio reciente que contrastaba la comprensión de oraciones en portugués e inglés en hablantes bilingües tardíos, se detectó un gran solapamiento de las áreas cerebrales activadas en ambas lenguas (Buchweitz, 2006). La escasa diferencia en la activación cerebral detectada en niveles de competencia y edad de adquisición puede explicarse en términos conocimiento explicito e implícito. En otras palabras, la competencia de una segunda lengua en una edad tardía puede haberse adquirido de una manera explicita, mediante un conocimiento declarativo, mientras que la competencia en una edad temprana se desarrolla de una manera implícita (automática), mediante un conocimiento implícito (Lebrun, 2002). En este sentido, parece que el conocimiento declarativo que posee el bilingüe menos competente no está del todo completado y, aunque actúa de manera similar al competente, no puede desarrollarlo del todo.

Además de estas dos demandas de procesamiento específico (competencia y edad de adquisición), otros estudios han puesto de manifiesto que también difiere el procesamiento en función del sistema de escritura. Por ejemplo, caracteres del alfabeto chino, también influyen en las respuestas cerebrales en tareas que requieren lectura de palabras en bilingües (véase Bolger et al., 2005; Tan et al., 2005). En este caso, el procesamiento de una segunda lengua requiere de un esfuerzo cognitivo extra de procesa- 
miento, los bilingües deben leer una palabra en diferentes sistemas de escritura adaptando, a la vez, diferentes rutas de significado. Dentro de un sistema alfabético en los que los lectores tienen que descifrar y relacionar sonidos con letras y combinaciones de letras (grafemas), utilizando para ello diferentes grados de regularidad del grafema al sonido en las distintas lenguas. En los sistemas logográficos, sin embargo, las palabras son probadas en el nivel de significado (morfemas). De manera diferente de los sistemas alfabéticos, los sistemas de escritura logográficas, los lectores deben procesar caracteres con información visual y espacial para acceder a su lexicón mental y a la información léxica y fonológica pertinente. Hay alguna evidencia empírica sobre este aspecto, como es el estudio desarrollado con fMRI por Buchweitz et al., (en preparación). En este trabajo participaron hablantes bilingües (japonésinglés) a los que se les instaba a leer oraciones completas en dos sistemas diferentes de escritura japonesa (uno de ellos logográfico llamado Kanji, y otro silábico, denominado Hiragana), además del inglés. Los resultados de este estudio corroboraron otros descubrimientos anteriores en los que los sistemas logográficos activaban áreas cerebrales asociadas a procesamiento visual y espacial, cuando se comparaba la misma lengua (el japonés) en sus dos sistemas de escritura (logográfico y silábico). En relación con el procesamiento de oraciones del L2 (en este caso el inglés), los resultados indicaron un esfuerzo cognitivo adicional más allá de las diferencias en el nivel de palabras entre el japonés e inglés.

En este sentido, algunos autores (e.g., Perani et al., 2005) han sugerido un punto de vista dinámico de la respuesta cerebral en el procesamiento de la L2. La activación cerebral de un lector bilingüe puede variar de acuerdo a demandas específicas de procesamiento. Pero a pesar de ello, puede afirmase que, de manera general, cuando los factores de edad de adquisición y competencia son controlados, los sujetos bilingües activan similares áreas corticales en las dos lenguas que hablan (Perani et al., 1998; Illes et al., 1999). Los estudios de neuroimagen indican que para un hablante bilingüe ambas lenguas son procesadas de manera similar, aunque no exactamente de la misma manera, localizándose en las mismas áreas cerebrales que se produce, incluso, en el caso de hablantes bilingües tardíos. En otras palabras, la competencia de procesamiento de una segunda lengua observada en la activación cerebral, es similar a la de la primera lengua.

La resonancia magnética funcional esta resultando ser una técnica muy válida para estudiar, entre otras cosas, la comprensión del lenguaje y el bilingüismo. Como cada hablante bilingüe desarrolla sus habilidades lingüísticas, el cerebro parece ser capaz de afinar con precisión los mecanismos cognitivos que participan en el procesamiento de diferentes lenguas. Quedan, al menos, dos cuestiones pendientes de investigar con esta técnica de neuroimagen. Por un lado, conocer la relación entre la carga de trabajo (workload) y la dificultad de los niveles de procesamiento y, por otra, analizar como se produce la habilidad del hablante bilingüe cuando cambia de una lengua a la otra tratando de comprender cualquiera de ellas.

\section{A modo de resumen}

Son varias las implicaciones que se derivan del papel que juega la causalidad en la cognición humana y su relación con los procesos de comprensión y coherencia del discurso. Dentro de estos puntos la propuesta teórica más interesante es la que nos muestra que la cognición causal es una característica inherente y, por tanto, universal del pensamiento humano: que la comprensión, como una actividad inteligente y muy relacionada con dicha cognición causal, requiere de un nivel de coherencia local y global con un fuerte contenido causal; y que para desarrollar tal actividad se requiere necesariamente de inferencias causales, que son las encargadas de tender puentes de conocimiento entre la información entrante y la que posee el lector, afín de establecer la coherencia (causal) necesaria en la comprensión final. Ahora bien, no puede olvidarse que la naturaleza de esta cognición causal está 'contextualizada' dentro de un marco 
cultural, social y lingüístico y que, como consecuencia de ello, los tipos de discurso que se desprenden de dicho contexto contienen diversas peculiaridades y atributos. Los tipos de discurso se han creado para dar respuesta a diferentes tipos de comunicación e información, conllevando una estructuración distinta. La cuestión que se ha debatido en este artículo es, precisamente, si los distintos tipos de discurso implican diferentes tipos de funcionamiento cognitivo que se cristalizan en diferentes formas de conexiones causales y, por tanto, conllevan diferentes tipos de inferencias causales, como también diferentes niveles de procesamiento. Asi mismo, si este comportamiento se ve o no influido por la lengua.

Una de las conclusiones a las que hemos llegado en este trabajo ha sido que la capacidad de generar inferencias es inherente a la naturaleza humana $y$, por consiguiente, de carácter universal, al igual que lo es la capacidad de generar relaciones causales, y ambas son relativamente independientes de un relativismo lingüístico y/o cultural. Ahora bien, también en este trabajo hemos puesto de manifiesto que los tipos de textos o de discurso, generan reacciones diferentes debidas, quizás, a su estructura misma. El tipo de estructura causal o la cantidad de inferencias que puedan generarse son variables sensibles al tipo de discurso. Esta es una acepción que se admite en buena parte de las culturas y de sus lenguas, lo que podemos considerar estos géneros de textos como transversales. De esta manera, concebimos el tipo de discurso como un tipo de relativismo discursivo, transversal e influyente en todas las lenguas estudiadas hasta ahora.

En referencia a los estudios más novedosos sobre neuroimagen y llevada a cabo en hablantes bilingües revelan una similitud en el procesamiento y en la activación cerebral de ambas lenguas relativamente independientes a la lengua que se hable. Cuando se han detectado algunas diferencias, estas son atribuibles al nivel de competencia del hablante respecto a su lengua materna, o a las diferencias en los sistemas de escritura en la que se presenta una determinada lengua, más que a un relativismo lingüístico propiamente dicho.
Una cuestión pendiente que queda abierta para el futuro es evaluar cómo estas diferencias quedan reflejadas en la activación cerebral. Esto es, si los tipos de inferencias o los tipos de pensamiento que se realizan bajo ambos tipos de discurso se localizan en zonas cerebrales diferentes. Resulta indudable que el presente y futuro de la investigación del discurso sobre estos temas va a aumentar en los próximos años, al verse reforzada por el avance de las tecnologías de la computación, así como por la integración de la neurociencia que, con el uso de técnicas que como el fMRI, va a permitir generar imágenes visuales de cómo la estructura del discurso que está siendo leído y procesado se representa en el cerebro mediante una imagen funcional. Con ayuda de esta técnica pueden ya identificarse cambios en la actividad cerebral cuando el lector trata de comprender un determinado discurso y esto nos puede ayudar a desvelar múltiples interrogantes como los aquí tratados.

\section{Referencias}

Aske, J. (1989). Path predicates in English and Spanish: A closer look. Proceedings of the Fifteenth Annual Meeting of the Berkeley Linguistics Society, 1-14.

Bartlett, F.C. (1932). Remembering. A study in experimental and social psychology. Cambridge. Cambridge University Press.

Barthes, R. (1966). Introduction to the Structural Analysis of the Narrative. Birmingham: Stencilled.

Bates, E., Devescovi, A. y Wulfeck, B. (2001). Annual Review of Psychology, 52, 369-396.

Berry, J.W. (2000). Cross-cultural psychology: A symbiosis of cultural and comparative approaches. Asian Journal of Social Psychology, 3, 197205.

Berry, J.W., Poortinga, Y.H., Segall, M.H. y Dasen, P.R. (1992). Cross-cultural psychology: Research and applications. Cambridge, MA: Cambridge University Press.

Bolger, D.J., Perfetti, C.A., Schneider, W., (2005). A 
cross-cultural effect on the brain revisited. Human Brain Mapping, 25, 91-104.

Brewer, W.R. (1980). Literary theory, rhetoric, and stylistics: Implications for psychology. En R.J. Spiro, B.C. Bruce y W.F. Brewer (Eds.), Theoretical issues in reading comprehension (pp. 221-239). Hillsdale, NJ: Lawrence Erlbaum Associates.

Britton, B.K. y Black, J.B. (Eds.) (1985). Understanding expository text: A Theoretical and Practical Handbook for Analyzing Explanatory Text. Hillsdale, NJ: Lawrence Erlbaum Associates.

Bruner, J.S. (1986). Actual minds, possible worlds. Cambridge, MA: Harvard University Press.

Buchweitz, A. (2006). Two languages, two input modalities, one brain: an fMRI study of Portuguese English bilinguals and Portuguese listening and reading comprehension effects on brain activation. Unpublished doctoral dissertation, Florianópolis: Universidade Federal de Santa Catarina.

Buchweitz, A., Mason, R.A., Hasegawa, M., y Just, M.A. (in preparation). Japanese and English sentence comprehension and writing systems: An fMRI study of first and second language effects on brain activation.

Candel, M., Fernández, C. y León, J.A. (2006). Una propuesta de evaluación de la competencia lectora en niños de $6^{\circ}$ de Primaria. Psicología Educativa, 12, 1, 65-78.

Chafe, W.L. (1980). The pear stories: Cognitive, cultural, and linguistic aspects of narrative production. Norwood, NJ: Ablex Publishing Company.

Cabeza, R. y Kingstone, A. (2006) Handbook of functional neuroimaging of cognition. Massachusetts: MIT press.

Danziger, E. (2001). Relatively speaking: Language, thought and kinship in Mopan Maya. Oxford Studies in Anthropological Linguistics. Oxford University Press.

de Vega, M. (1995). Backward updating of mental models during continuous reading of narratives.
Journal of Experimental Psychology: Learning, Memory, and Cognition, 21, 373-385.

Dewey, J. (1938). Logic. The theory of inquiry. Nueva York: Holt.

Einstein, G.O., McDaniel, M.A., Owen, P.D. y Coté, N.C. (1990). Encoding and recall of texts: The importance of material appropriate processing. Journal of Memory and Language, 29, 566581.

Escudero, I. (2004). Procesamiento de inferencias elaborativas en la comprensión del discurso y según el tipo de texto. Tesis doctoral inédita. Universidad Autónoma de Madrid.

Escudero, I. y León, J.A. (2007). Procesos inferenciales en a comprensión del discurso escrito. Influencia de la estructura del texto en los procesos de comprensión. Revista Signos, 40, 311-336.

Escudero, I. (2010). Las influencias en la comprensión lectora: una ventana hacia los procesos cognitivos en segundas leyes. Revista Nebrija de Lingüística Aplicada a la Enseñanza de Lenguas, 7, 1-32.

Escudero, I. y León, J.A. (en revisión). Discourse comprehension processes between types of texts: A cross-cultural/cross-language study. Journal of Cross-Cultural Psychology.

Foley, W. (1997). Anthropological Linguistics: An Introduction. Blackwell Publishers.

Gentner, D. y Goldin-Meadow, S. (2003). Language in mind: Advances in the study of language and thought. Cambridge, MA: MIT Press.

Glenberg, A.M., Meyer, M. y Lindem, K. (1987). Mental models contribute to foregrounding during text comprehension. Journal of Memory and Language, 26, 69-83.

Goldman, S.R. y Bisanz, G.L. (2002). Toward a functional analysis of scientific genres: Implications for understanding and learning processes. En J. Otero, J.A. León y A.C. Graesser (Eds.), The psychology of science text comprehension (pp. 19-50). Hillsdale, NJ: Lawrence Erlbaum Associates.

Goldman, S.R., Varma, S. y Coté, N. (1996). Extending capacity-constrained construction inte- 
gration: toward "smarter" and flexible models of text comprehension. En B.K. Britton y A.C. Graesser (Eds.), Models of understanding text (pp. 73-113). Hillsdale, NJ: Lawrence Erlbaum Associates.

Graesser, A.C. (1981). Prose comprehension beyond the world. Nueva York: Springer-Verlag.

Graesser, A.C. y Bertus, L.B. (1998). The construction of causal inferences while reading expository texts on science and technology. Scientific Studies of Reading, 2, 247-269.

Graesser, A.C. y Bower, G.H. (Eds.) (1990). Inferences and text comprehension. San Diego, CA: Academic Press.

Graesser, A.C., León, J.A. y Otero, J.C. (2002). Introduction to the psychology of science text comprehension. En J.C. Otero, J.A. León y A.C. Graesser (Eds.), The psychology of science text comprehension (pp. 1-15). Mahwah, NJ: Lawrence Erlbaum Associates.

Graesser, A.C., Swamer, S.S., Baggett, W.B. y Sell, M.A. (1996). New models of deep comprehension. En B.K. Britton y A.C. Graesser, (Eds.), Models of understanding text (pp. 1-32). Mahwah, NJ: Lawrence Erlbaum Associates.

Gumperz, J. y Levinson, S. (1996). Rethinking Linguistic Relativity. Cambridge, UK: Cambridge University Press.

Harris, J.L., Rogers, W.A. y Qualls, C.D. (1998). Written language comprehension in younger and older adults. Journal of Speech, Language and Hearing Research, 41, 603-617.

Hoerl, C. y McCormack, T. (2001). Time and memory: Issues in philosophy and psychology. Oxford, UK: Oxford University Press.

Illes, J., Francis, W. S., Desmond, J.E., Gabrieli, J.D.E., Glover, G.H., Poldrack, R., Lee, C.J., Wagner, A.D. (1999). Convergent cortical representation of semantic processing in bilinguals. Brain and Language, 70, 347-363.

Johnson-Laird, P.N. (1983). Mental models: Towards a cognitive science of language, inference, and consciousness. Cambridge: Cambridge University Press.
Kim, K.H.S., Relkin, N.R., Lee, K. y Hirsch, J. (1997). Distinct cortical areas associated with native and second languages. Nature, 388, 171174.

Kim, U., Park, Y.-S. y Park, D. (2000). The challenge of cross-cultural psychology: The role of the indigenous psychologies. Journal of CrossCultural Psychology, 31, 63-75.

Kucan, L. y Beck, I.L. (1996). Four fourth graders thinking aloud: An investigation of genre effects. Journal of Literacy Research, 28, 259-287.

Labov, W. y Waletzsky, J. (1967). Narrative analysis: Oral versions of personal experience. En J. Helm (Ed.), Essays in the verbal and visual arts (pp. 1244). Seattle: University of Washington Press.

Langston, M. y Trabasso, T. (1999). Modeling causal integration and availability of information during comprehension of narrative texts. En $\mathrm{H}$. van Oostendorp y S.R. Goldman (Eds.), The construction of mental representations during reading (pp. 29-69). Mahwah, NJ: Lawrence Erlbaum Associates.

Lebrun, Y. (2002). Implicit competence and implicit knowledge. In: F. Fabbro (Ed.) Advances in the neurolinguistics of bilingualism, Udine: Forum, pp. 299-313.

León. J.A. (1986). La memoria de los niños a través de los cuentos: Un análisis experimental. Mérida: Universidad Nacional de Educación a Distancia.

León, J.A. (Coord.) (2003). Conocimiento y discurso. Claves para inferir y comprender. Madrid: Pirámide.

León, J.A. (2004). Adquisición de conocimiento y comprensión. Origen, evolución y método. Madrid: Biblioteca Nueva.

León, J.A. (2004). ¿Por qué las personas no comprenden lo que leen? Psicología Educativa, 10, 101-116.

León, J.A. (2009). Neuroimagen de los procesos de comprensión en la lectura y el lenguaje. Psicología Educativa, 15, 61-71.

León, J.A., Escudero, I., Olmos, R., Sanz, M. Dávalos, T. y García, T. (2009). ECOMPLEC: Un Modelo de Evaluación de la Comprensión 
Lectora en Diversos Tramos de la Educación Secundaria, (2009), Psicología Educativa, 15, 123-142.

León, J.A., Escudero, I. y van den Broek, P. (2003). La influencia del género del texto en el establecimiento de inferencias elaborativas. En J.A. León (Coord.), Conocimiento y discurso. Claves para inferir y comprender (pp. 153-170). Madrid: Pirámide.

León, J.A., Hytönnen, K. y Escudero, I. (en preparación). The influence of genre on the activation of elaborative inferences: A cross-cultural study based on thinking-aloud tasks.

León, J.A., Otero, J.C., Escudero, I., Campanario, J.M. y Pérez, O. (1999, Julio). Levels of causal explanations in psychology and physics domains. An expert/novice study. Comunicación presentada en el VI European Congress of Psychology. Roma, Italia.

León, J.A. y Peñalba, G.E. (2002). Understanding causality and temporal sequence in scientific discourse. En J.C. Otero, J.A. León y A.C. Graesser (Eds.), The psychology of science text comprehension (pp. 199-221). Mahwah, NJ: Lawrence Erlbaum Associates.

León, J.A. y Slisko, J. (2000). La dificultad comprensiva de los textos de ciencias. Nuevas alternativas para un viejo problema educativo. Psicología Educativa, 6, 7-26.

Lucy, J. (1992). Grammatical categories and Cognition: a Case Study of the Linguistic Relativity Hypothesis. Cambridge, UK: Cambridge University Press.

Mahendra, N., Plante, E., Magloire, J., Milman, L. y Trouard, T.P. (2003). fMRI variability and the localization of languages in the bilingual brain. NeuroReport, 14, 1225-1228.

Marian, V., Shildkrot, Y., Blumenfeld, H.K., Kaushanskaya, M., Faroqi-Shah, Y. y Hirsch J. (2007). Cortical activation during word processing in late bilinguals: similarities and differences as revealed by functional magnetic resonance imaging. Journal of Clinical and Experimental Neuropsychology 29, 247-65.
McDaniel, M.A., Einstein, G.O., Dunay, P.K. y Cobb, R.E. (1986). Encoding difficulty and memory: Toward a unifying theory. Journal of Memory and Language, 25, 545-656.

McKeown, M.G., Beck, I.L., Sinatra, G.M. y Loxterman, J.A. (1992). The contribution of prior knowledge and coherent text to comprehension. Reading Research Quarterly, 27, 78-93.

Mandler, J.M. y Johnson, N.S. (1977). Remembrance of things parsed: Story structure and recall. Cognitive Psychology, 9, 111-151.

Mandler, J.M., Scribner, S., Cole, M., y De Forest, M. (1980). Cross-cultural invariance in story recall. Child development, 51, 19-26.

Martins, I. (2002). Visual Imagery in School Science Texts. En J.C. Otero, J.A. León y A.C. Graesser (Eds.), The psychology of science text comprehension (pp. 73-90). Mahwah, NJ: Lawrence Erlbaum Associates.

McDaniel, M.A., Einstein, G.O., Dunay, P.K. y Cobb, R.E. (1986). Encoding difficulty and memory: Toward a unifying theory. Journal of Memory and Language, 25, 545-656.

Millis, K.K. y Graesser, A.C. (1994). The timecourse of constructing knowledge-based inferences for scientific texts. Journal of Memory and Language, 33, 583-599.

Morris, M.W., Nisbett, R.E. y Peng, K. (1995). Causal attribution across domains and cultures. En D. Sperber, D. Premack y A.J. Premack (Eds.), Causal cognition: A multidisciplinary debate (pp. 577-614). Nueva York: Clarendon Press/Oxford University Press.

Ohlsson, S. (2002). Generating and understanding qualitative explanations. En J.C. Otero, J.A. León y A.C. Graesser (Eds.), The psychology of science text comprehension (pp. 91-128). Mahwah, NJ: Lawrence Erlbaum Associates.

Otero, J.C., León, J.A. y Graesser, A.C. (Eds.) (2002). The psychology of science text comprehension. Mahwah, NJ: Lawrence Erlbaum Associates.

Paradis, M. (2003). Differential use of cerebral mechanisms in bilinguals. En: M.T. Banich, M. 
Mack (Eds.), Mind, Brain, and Language: Multidisciplinary perspectives. Lawrence Erlbaum, pp. 351-370.

Perani, D., y Abutalebi, J. (2005). The neural basis of first and second language processing. Current Opinion in Neurobiology, 15, 202-206.

Perani, D., Paulesu, E., Galles, N.S., Dupoux, E., Dehaene, S., Bettinardi, V., Cappa, S.F., Fazio, F. y Mehler, J. (1998). The bilingual brain: proficiency and age of acquisition of the second language. Brain, 121, 1841-1852.

Piaget, J. (1927a). La causalité physique chez l'enfant [The child's conception of physical causality]. París: Alcan.

Piaget, J. (1927b). L'explication de l'ombre chez l'enfant (Children's explanations of shadows). Journal de Psychologie, 24, 230-242.

Pinker, S. (1994). The language instinct: How the mind creates language. New York, NY: Morrow.

Polkinghorne, D.E. (1988). Narrative knowing and the human sciences. Nueva York: State University of New York Press.

Ricoeur, P. (1998). Tiempo y narración. Configuración del tiempo en el relato histórico. Madrid: Siglo veintiuno (Trabajo original publicado en 1985).

Rosenbaum, D.A. y Collyer, C.E. (Eds.) (1998). Timing of behavior: Neural, psychological, and computational perspectives. Cambridge, MA, US: The MIT Press.

Rumelhart, D.E. (1975). Notes on a schema for stories. En D.G. Bobrow y A. Collins (Eds.), Representation and understanding: Studies in cognitive science (pp. 211-236). Nueva York: Academic Press.

Salmon, W.C. (1998). Causality and explanation. Oxford, UK: Oxford University Press.

Sapir, E. (1921). Language. New York, NY: Harcout, Brace, and World.

Schank, R.C. (1975). The role of memory in language processing. En C. Cofer y R. Atkinson (Eds.), The nature of human memory. San Francisco: Freedman. (Trad. Cast.: Estructura de la memoria humana. Barcelona: Omega, 1979).
Schank, R.C. y Abelson, R.P. (1977). Scripts, plans, goals and understanding: An inquiry into human knowledge structures. Hillsdale, NJ: Lawrence Erlbaum Associates.

Singer, M., Harkness, D. y Stewart, S.T. (1997). Constructing inferences in expository text comprehension. Discourse Processes, 24, 199-228.

Slobin, D. I. (1987). Thinking for speaking. Proceedings of the Thirteenth Annual Meeting of the Berkeley Linguistics Society, 435-444.

Slobin, D. I. (1996). Two ways to travel: Verbs of motion in English and Spanish. En M. Shibatani y S. A. Thompson (Eds.), Grammatical constructions: Their form and meaning (pp. 195-217). Oxford: Oxford University Press.

Slobin, D. I. (1997). Mind, code, and text. En J. Bybee, J. Haiman, y S. A. Thompson (Eds.), Essays on language function and language type: Dedicated to T. Givón (pp. 437-467). Amsterdam/Philadelphia: John Benjamins.

Slobin, D.I. (2003). Language and thought online: Cognitive consequences of linguistic relativity. En D. Gentner y S. Goldin-Meadow (Eds.), Language in mind: Advances in the study of language and thought (pp. 157-192). Cambridge, MA: MIT Press.

Slobin, D. I., y Hoiting, N. (1994). Reference to movement in spoken and signed languages: Typological considerations. Proceedings of the Twentieth Annual Meeting of the Berkeley Linguistics Society, 487-505.

Sperber, D., Premack, D. y Premack, A.J. (Eds.). (1995). Causal cognition: A multidisciplinary debate. Nueva York: Clarendon.

Stein, N.L. y Glenn, C.G. (1979). An analysis of story comprehension in elementary school children. En R.O. Freedle (Ed.), New directions in discourse processing (pp. 53-120). Hillsdale, NJ: Lawrence Erlbaum Associates.

Tan, L.H., Laird, A.R., Li, K., Fox, P.T. (2005). Neuroanatomical correlates of phonological processing of Chinese characters and alphabetic words: A meta-analysis. Human Brain Mapping, 25, 83-91. 
Tannen, D. (1980). A comparative analysis of oral narrative strategies: Athenian Greek and American English. En W.L. Chafe (Ed.), The pear stories: Cognitive, cultural, and linguistic aspects of narrative production. Norwood, NJ: Ablex Publishing Company.

Tannen, D. (1983). "I take out the rok-dok!": How Greek women tell about being molested (and create involvement). Anthropological Linguistics, 359-374.

Tannen, D. (1984). Spoken and written narrative in English and Greek. En D. Tannen (Ed.), Coherence in spoken and written discourse. Norwood, NJ: Ablex Publishing Company.

Tannen, D. (1988). Hearing voices in conversation, fiction, and mixed genres. En D. Tannen (Ed.), Linguistics in contexts: Connecting observation and understanding. Norwood, NJ: Ablex Publishing Company.

Thorndyke, P.W. (1977). Cognitive structures in comprehension and memory of narrative discourse. Cognitive Psychology, 9, 77-110.

Trabasso, T., Secco, T. y van den Broek, P. (1984). Causal cohesion and story coherence. En H. Mandl, N.L. Stein y T. Trabasso (Eds.), Learning and comprehension of text (pp. 83-111). Hillsdale, NJ: Lawrence Erlbaum Associates.

Trabasso, T. y Sperry, L.L. (1985). The causal basis for deciding importance of story events. Journal of Memory and Language, 24, 595-611.

Trabasso, T., van den Broek, P.W. y Suh, S.Y. (1989). Logical necessity and transitivity of causal relations in stories. Discourse Processes, $12,1-25$. van den Broek, P. (1988). The effects of causal relations and hierarchical position on the importance of story statements. Journal of Memory and Language, 27, 1-22.

van den Broek, P. (1989). The effects of causal structure on the comprehension of narratives: Implications for education. Reading Psychology, 10, 19-44.

van den Broek, P. (1990). Causal inferences and the comprehension of narrative text. En A.C. Graesser y G.H. Bower (Eds.), Inferences and text comprehension (pp. 175-196). San Diego, CA: Academic Press.

van den Broek, P., Virtue, S., Gaddy, M., Tzeng, Y. y Sung, Y. (2002). Comprehension and memory of science texts: Inferential processes and the construction of a mental representation. En J.C. Otero, J.A. León y A.C. Graesser (Eds.), The psychology of science text comprehension (pp. 131154). Mahwah, NJ: Lawrence Erlbaum Associates.

van den Broek, P., Young, M., Tzeng, Y. y Linderholm, T. (1999). The landscape model of reading: Inferences and the online construction of a memory representation. En H. van Oostendorp y S. Goldman (Eds.), The construction of mental representations during reading (pp. 71-98). Mahwah, NJ: Lawrence Erlbaum Associates.

van Dijk, T. y Kintsch, W. (1983). Strategies of discourse comprehension. Nueva York: Academic Press.

Whorf, B. (1956). Language, Thought, and Reality: Selected Writings of Benjamin Lee Whorf, edited by Carroll JB.

Manuscrito recibido: 14/07/2009

Revisión recibida: 02/09/2009

Manuscrito aceptado: 29/09/2009 